\title{
Effects of Methylene Blue Mediated Photodynamic Therapy on Sol- idEhrlich Tumor and Second Ehrlich Tumor Implant in Mice
}

\author{
MuriloPenteado Del-Grande, Lucas Martins Chaible, Maria Lucia ZaidanDagli"
}

Laboratory of Experimental and Comparative Oncology, Department of Pathology, School of Veterinary Medicine and Animal Science, University of São Paulo, São Paulo, SP, Brazil.

"Corresponding author: Maria Lucia ZaidanDagli, Laboratory of Experimental and Comparative Oncology, Department of Pathology, School of Veterinary Medicine and Animal Science, University of São Paulo, São Paulo, SP, Brazil; Tel: +00-55 11 3091 7712; Fax:+00-55 113091 7829; E-mail: mlzdagli@usp.br

Citation: Del-Grande MP, Chaible LM, ZaidanDagli ML (2017) Effects of Methylene Blue Mediated Photodynamic Therapy on Solid Ehrlich Tumor and Second Ehrlich Tumor Implant in Mice. Arch Vet Sci Technol, 2017: AVST-122. DOI:10.29011/AVST$122 / 100022$

Received Date: 20 June, 2017; Accepted Date: 21 June, 2017; Published Date: 28 June, 2017

\begin{abstract}
PhotoDynamic Therapy (PDT) uses interaction of light, photosensitizing agent and production of reactive oxygen species, leading to cell death. Methylene Blue (MB) issued for PDT due to its photochemical properties. Here we investigated theeffects of Methylene Blue Mediated Photodynamic Therapy (MB-PDT) on the solidEhrlich tumor and second Ehrlich tumor implant in mice. For MB-PDT, MB at 1\%and diode laser were used. Swiss male mice received dorsal inoculation of Ehrlichtumor cells and, 9 days after, mice were separated into 3 groups: MB-PDT (Group1) surgically removed (Group 2), or untreated (Group 3). One day after treatment,all groups received a second Ehrlich tumor implant on the left footpad, measured for 17 days. Spleen, lymph nodes and tumor mass were weighed upon necropsy andprocessed for histopathology. Group 1 Ehrlich tumors showed significant sizereduction after MB-PDT. Morphometry of second Ehrlich tumor in Group 1 miceshowed significantly lower volume fraction of tumor cells, higher inflammatoryinfiltrate and necrosis. Relative spleen weight was higher in Group 1 mice, withwhite pulp hyperplasia. Per these results, MB-PDT reduced primary Ehrlich tumorgrowth and impacted the growth of a second tumor. These results point towardspossible biotechnological applications of MB-PDT.
\end{abstract}

Keywords:Photodynamic Therapy, Methylene Blue, Ehrlich Tumor, CancerTreatment.

\section{Introduction}

Current treatment of solid tumors is mainly based on their surgical excision.Radiotherapy and chemotherapy are adjuvant treatment modalities commonly used,depending on tumor type. PhotoDynamic Therapy (PDT) is a method to treat cancer based on theinteraction between a photosensitizing agent, light and molecular oxygen. There arenumerous types of photosensitizers, which can be administered by intravenous, oralor topical routes. The illumination of the tumor with visible light in order to activatethe photosensitizer is taken after certain time of agent administration, leading toproduction of Reactive Oxygen Species (ROS), determining cellular death by necrosisand/or apoptosis [1]. Methylene Blue (MB) is a molecule that has been consideredas a drug for photodynamic therapy (PDT). According to [2], MB has the potential to treat a variety of cancerous and non-cancerous diseases,with low toxicity and no side effects. An interesting feature of PDT is that it may activate the immune systemagainst tumor cells. The phototoxic effects on cell membrane release a number ofinflammatory mediators leading to innate immune system activation. Localinflammation and neoplastic cell death provoke tumor antigen presentation[3]. Antigenpresentation promotes activation of adaptive immune system and there issensitization of $\mathrm{T}$ lymphocytes $(\mathrm{CD} 8+)$ leading to a specific response to the treatedtumor. This adaptive immune response would be involved in the killing of tumorcells. In addition, B lymphocytes are sensitized to tumor antigens, promoting a long-termtumor control. The production of antitumor antibodies may be suggested,although this has not yet been shown through experimental modelsin this study[4], we aimed to verify if treating a primary Ehrlich tumor with Methylene Blue (MB) at 1\% based PDT (MB-PDT) could present any systemicinfluence on the growth and behavior of a second Ehrlich tumor implant in mice. 


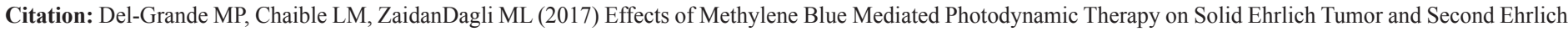
Tumor Implant in Mice. Arch Vet Sci Technol, 2017: AVST-122.

\section{Materials and Methods}

\section{Animals}

Male Swiss mice, weighing 25-35g, were obtained from the Animal Facility ofthe Department of Pathology, School of Veterinary Medicine and Animal Science,University of São Paulo, Brazil. During the experiment, animals were kept inpolycarbonate boxes covered with wood shavings, kept in a room with ventilation,exhaust and controlled climate with temperatures between 22 and $24{ }^{\circ} \mathrm{C}$, relativehumidity $55 \%$ and light night/day cycle of 12 hours. Animals were fed with abalanced diet and water ad libitum. Animal use was in accordance with the ethicalprinciples of animal experimentation of the Ethics Committee on Animal Use of theSchool of Veterinary Medicine and Animal Science of the University of Sao Paulo,protocol number 2235/2011.

\section{Experimental Design}

Thirty Swiss male mice were inoculated with Ehrlich tumor cells on thesubcutaneous region of the dorsum. Nine days after, Group 1 mice $(\mathrm{n}=10)$ receivedone session of MB-PDT (described ahead), and Group 2 mice $(\mathrm{n}=10)$ weresubmitted to surgical excision of primary tumor. A third group of mice, Group 3(n=10) received no treatment and primary tumor kept growing. Twenty-four hoursafter MB-PDT, or surgery of primary tumor, Group 1, Group 2 and Group 3 mice5received a second Ehrlich tumor implant on the left footpad; these tumors weremeasured with a caliper for 17 days, when mice were euthanized [5]. At necropsy,spleen and lymph nodes were weighed and processed for histological analysis. Tumor samples were collected, routinely processed for embedding in paraffin, andthe $5 \mu \mathrm{m} \mathrm{H \& E}$ sections were analyzed trough quantitative morphometry, according to[6]. In addition, blood samples were takenfor leukocyte count before euthanasia.

\section{Ehrlich Tumor Inoculation}

Animals were physically restrained and with sterile syringes and needleswere given $0.05 \mathrm{ml}$ of suspension containing $2.5 \times 106$ tumor cells on thesubcutaneous dorsal region (primary Ehrlich tumor) or left footpad (second Ehrlichtumor).

\section{Ehrlich Tumor Treatment}

\section{Group 1-Methylene Blue Photodynamic Therapy}

At ninth day of primary tumor growth, the Group 1 animals received onesession of MB-PDT. Animals were anesthetized with intraperitoneal administrationofketamine hydrochloride $(100 \mathrm{mg} /$ $\mathrm{kg}$ ) associated with xylasin $(20 \mathrm{mg} / \mathrm{kg})$. Thetumor site was shaved and local antisepsis was performed with iodized $70^{\circ}$ alcohols. With sterile needles and syringes, $0.05 \mathrm{ml}$ of $1.0 \%$ methylene blue solution $\left(\right.$ Synth $\left.{ }^{\circledR}\right)$ in sterile $0.9 \% \mathrm{NaCl}$ was administered by intratumoral injection. After 10 minutes, tumor was irradiated for 9 minutes at a dose of $54 \mathrm{~J}$, using an InGaAIP medium diode laser emitting visible red light at a wavelength of $660 \mathrm{~nm}$ and power output of $100 \mathrm{~mW}$. During irradiation, all researchers wore safety goggles.

\section{Group 2-Surgical Excision}

At ninth day of primary tumor growth, the dorsal Ehrlich tumors from Group 2animals were surgically excised. Animals were anesthetized with intraperitonealadministration of ketamine hydrochloride $(100 \mathrm{mg} / \mathrm{kg})$ associated with xylasin $(20 \mathrm{mg} / \mathrm{kg})$. The tumor site was shaved and local antisepsis performed with iodized $70^{\circ}$ alcohols. The tumors were surgically excised, with margins of approximately $2 \mathrm{~mm}$.Skin sutures were performed with nylon (4-0) in simple uninterrupted sutures.Quantitative morphometric analysis of the second Ehrlich tumorVolumetric Fraction (VF) of footpad cellular componentsImages of $\mathrm{H} \& \mathrm{E}$ sections of the left

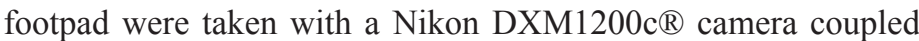
to a light microscope Nikon Eclipse E800®. With 20xmagnification lenses, 8 fields were photographed and images were digitized byIMAGE PRO PLUS ${ }^{\circledR}$ software. Using open source ImageJ software, a grid with 35points was overlapped on each field. Each point was determined as tumor cells, inflammatory infiltrate, necrosis and other (Vessels, Muscle, Subcutaneous, etc.).

\section{Footpad Necrosis Area}

Images of H\&E sections of the left footpad were taken with a Nikon DXM $1200 c^{\circledR}$ camera coupled to a light microscope Olympus model SZX2-ILLK ${ }^{\circledR}$. With 0,25xmagnification lenses, the entire footpad area was photographed using opensource ImageJ software, total area and necrosis area were determined.

\section{Statistical Analysis}

For data analysis, the statistical software Minitab $16^{\circledR}$ (Minitab Inc., PA, USA) and GraphPad Prism5.00 ${ }^{\circledR}(\mathrm{GraphPad}$ Software, Inc., San Diego, USA) wereused. Normality was verified using the Anderson-Darling test. Homoscedasticity wasverified by Bartlett test. For parametric data, we used analysis of variance ANOVAfollowed by the Tukey's test for comparison between experimental groups. Kruskal-Wallis test followed by Dunn's test for comparison between groups was used fornon-parametric data. The growth curve of the Ehrlich tumor was analyzed by twowayANOVA. Differences of $p<0.05$ were considered statistically significant. Datawere expressed as mean \pm standard deviation.

\section{Results}

Analysis of Group 1 Ehrlich tumor growththe primary Ehrlich tumor inoculated at the dorsum of Group 1 animals, which received MB-PDT, presented ulceration and necrosis; the epithelium healedin a few days. Therefore, the tumors were not completely cured, and continued togrow. The Group 2 animals, which received surgical excision of primary tumor, didnot show any signs of local recurrence, with no further primary tumor growth.The untreated 


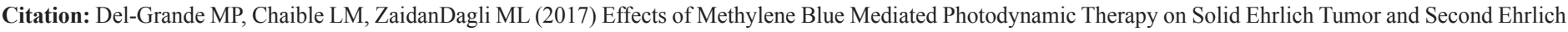
Tumor Implant in Mice. Arch Vet Sci Technol, 2017: AVST-122.

animals of Group 3 showed continuous primary tumor growthto the end of the experiment.

Analysis of Second Ehrlich Tumor Growthas previously stated, 24 hours after the initial treatment, animals from Group1, Group 2 and Group 3 were inoculated with second Ehrlich tumor cells on the leftfootpad, and the tumor growth was evaluated with a caliper for 17 days. (Figure 1)shows the left footpad growth curve (diameter in $\mathrm{mm}$ ) by time (days) of mice bearingEhrlich solid tumor treated either with PDT, surgery or no treatment (control). Dataare presented as mean \pm standard deviation. There was no significant differencebetween groups PDT and Surgery and Control $(\mathrm{p}>0.05)$.

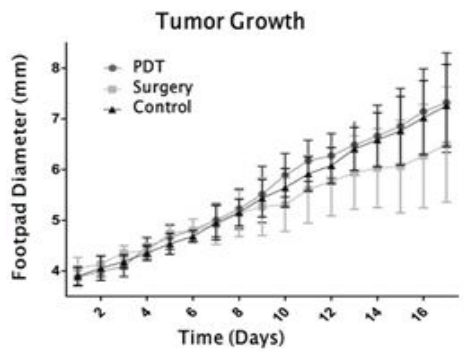

Figure 1: Ehrlich tumor growth in mouse left footpad (diameter in $\mathrm{mm}$ ) by time (days)of mice bearing Ehrlich Solid tumor treated either with MB-PDT (Group 1), surgery(Group 2) or no treatment (Control-Group 3). Data are presented as mean \pm standard deviation. There was no significant difference between groups (two-way ANOVA p > 0.05).

\section{Quantitative Morphometric Analysis}

\section{Volumetric Fraction of Footpad Cellular Components: Tumor Cells and Inflammatory Infiltrate}

The second tumor transplant was evaluated through morphometric analysisusing images of H\&E sections of the left footpad. (Figure 2)

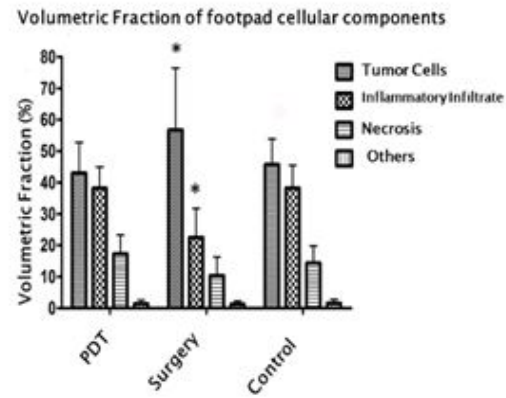

Figure 2: Volume Fraction (VF) of the cellular components in Ehrlich tumor infootpads of mice. The ANOVA revealed a significant difference in VF of tumor cells $(\mathrm{p}<0.0001)$ and inflammatory infiltrate $(\mathrm{p}<0.0001)$. The Turkey post-test showedthat VF of tumor cells is significantly higher in the surgery group $(\mathrm{p}<0.05)$, with a VF of inflammatory infiltrate significantly lower in this group ( $\mathrm{p}<0.05)$. The VF of necrosis showed no significant difference between groups $(\mathrm{p}=0.0669)$.

\section{Footpad Necrosis Area}

The necrotic areas in the left footpad were measured and the results areshown in (Figure 3). Using the Kruskal-Wallis test the results are significantdifferences in the necrotic area in different groups $(\mathrm{p}=0.0007)$. The Dunn testshowed a significantly smaller area of necrosis $(\mathrm{p}<0.05)$ in the surgery groupcompared with PDT and control groups.

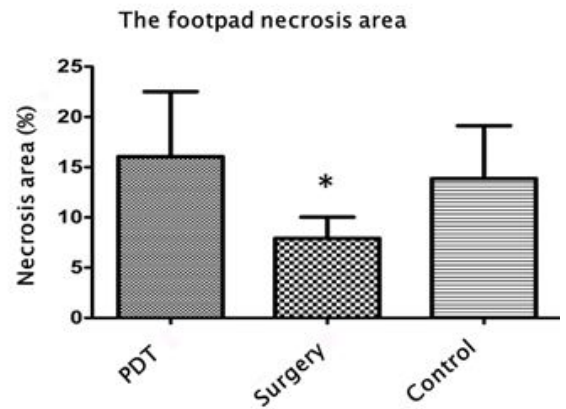

Figure 3: Necrotic area within solid Ehrlich tumor in the left footpad of mice. TheKruskal-Wallis test revealed a significant difference in the necrotic area on differentgroups (Groups 1, 2 and 3) $(p=0.0007)$. The Dunn test showed a significantlysmaller area of necrosis $(p<0.05)$ in the surgery group compared with MB-PDT andcontrol groups.

\section{Lymphoid Organ Analysis}

\section{Relative Spleen Weight}

(Figure 4)illustrates data on relative spleen weight. The ANOVA revealedsignificant difference between groups $(p<0.0001)$. The Tukey post test showed thatrelative spleen weight of the surgery group (Group 2) was significantly lower $(\mathrm{p}<0.05)$ when compared to PDT (Group 1) and control (Group 3) groups.

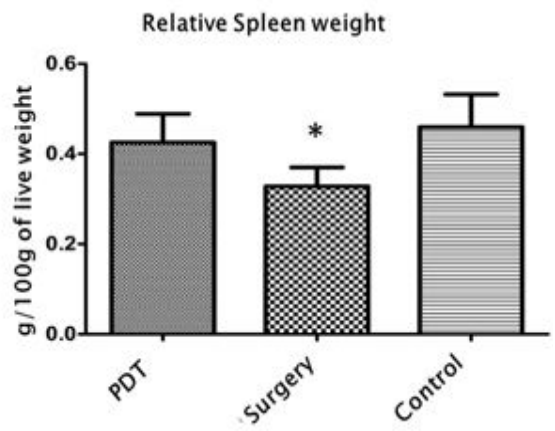

Figure 4: Relative spleen weight of mice bearing Ehrlich tumor and treated with MB18PDT (Group 1) or surgery (Group 2). The ANOVA revealed significant differencesbetween groups $(\mathrm{p}<0.0001)$. The Turkey post test showed that the relative spleenweight of the surgery group was significantly lower $(\mathrm{p}<0.05)$ compared with MBPDTand control groups.

\section{Spleen Histological Analysis}

(Figure 5) illustrates histological pattern found in mice bearing Ehrlich solidtumor spleen. Surgery group spleens (Group 2) did not show any significantmorphological changes (Figure 5B). Group 


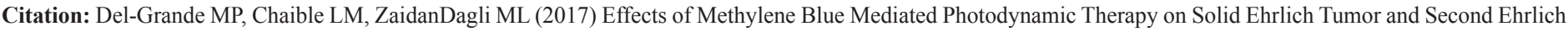
Tumor Implant in Mice. Arch Vet Sci Technol, 2017: AVST-122.

1 (MB-PDT) (Figure 5A) and Group 3(control group) (Figure 5C) mice revealed organ architecture preservation; however,lymphoid follicles and its germinal center and marginal zone had apparently becomemore prominent, with coalescent areas between follicles. Findings suggest lymphoidhyperplasia in the spleen of Group 1 (MB-PDT) animals.
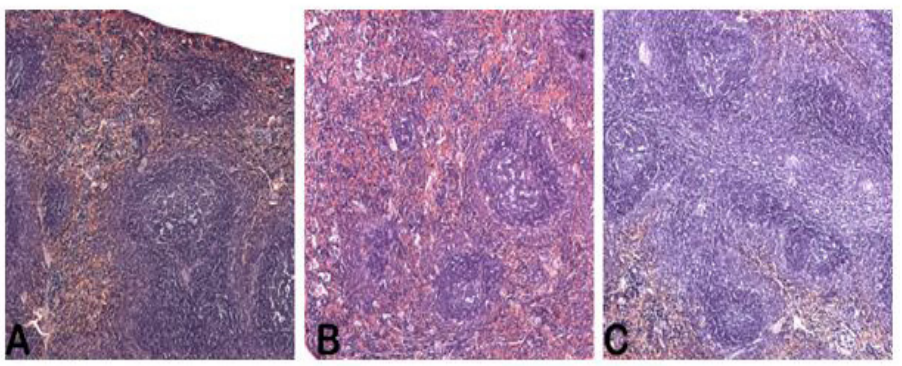

Figure 5: Histological pattern in the spleen of mice bearing Ehrlich solid tumor andtreated with MB-PDT (Group 1) or surgery (Group 2). Surgery group didn't show anysignificant morphological changes (Figure 5A). MB-PDT (Group 1) (Figure 5B) andcontrol group (Group 3) (Figure 5C) mice revealed preserved organ architecture,however lymphoid follicles, their germinal centers and marginal zone had apparentlybecome more prominent, with coalescent areas between follicles. It has also beenobserved an expansion of the cellular cuff around the central arteries. The findingssuggest a lymphoid hyperplasia in the spleen of these animals.

\section{Relative Lymph Node Weight}

The ANOVA regarding the relative weight of the left popliteal lymph noderevealed no significant differences between groups $(\mathrm{p}=0.0622)$.

\section{Left Popliteal Lymph Node Histological Analysis}

Histological sections of popliteal lymph nodes showed similar patternsbetween groups. There was marked hyperplasia of lymphoid follicles and germinalcenter increase. There were many mononuclear cells phagocyting cellular debris( tangible body macrophages) in lymphoid follicles. The pattern is characteristic ofreactive lymph node hyperplasia. Para cortical and interfollicular region hyperplasia were observed and caused by increase of lymphoid cells with eccentric nuclei, similar to plasma cells. There was also sinusoidal histiocytosis characterized bymedullary cord hyperplasia and hyper cellularity of medullary sinuses, filled withreticular cells. All groups had cells with the phenotypic characteristics of the Ehrlichtumor, including atypical mitoses. Those cells were observed in the sub capsular and interfollicular regions, although no large tumor proliferation foci that would characterize metastasis were found.

\section{Blood Leukocyte Count}

The ANOVA for leukocyte count revealed no significant differences betweengroups (data not shown).

\section{Discussion}

In this study, we aimed to verify if treating primary tumor with MB-PDT couldpresent any influence on the growth and behavior of a second tumor. For thispurpose, a model of subsequent implants of Ehrlich tumor was used.While the treatment of the solid tumor with MB-PDT caused necrosis in Group1 mice, the footpad tumor growth curve analysis showed no significant differenceamong experimental groups 1, 2 or 3 , during the 17-day measurement. It is possibleto see a trend on Group 1 approaching the Group 3 group from day 13. The finalmean diameter of the surgery group is below the others, but comparing only thisfinal diameter, there was no statistically significant difference.

The diameter increases of the footpad inoculated with Ehrlich solid tumor ismainly due to the proliferation of tumor cells, but the inflammatory reaction is alsoinvolved in increasing tumor size. According to literature, PDT induces adaptiveimmunity against treated tumor and neoplastic cells that did not die duringtreatment. Tumor immunity is systemic, long-term and does not happen only onprimary tumor. The response is observed against metastasis and recurrence ofspontaneous tumors. This was also seen on experimental models withtransplantable cells inoculated at distant site from the primary tumor.Treated fibro sarcomas MS-2 in mice with CASPc photo sensitizer and diode laser with an irradiation scheme similar to the present study[7]. There was no difference in the survival rate when compared to surgicaltreatment, but when animals received a second tumor challenge, surgically treatedanimals soon died while those treated with PDT survived for up to 100 days[8] used BALB/c mice and transplantable tumors(Colon 26 and EMT6). PDT was performed with $\mathrm{HpD}$ and Argon laser. These tumorsproduce lung metastases but those diminished after 10 days on PDT treatedanimals when compared to surgically treated mice. In the present experimental model, a difference among the growth curve ofanimals previously treated with MB-PDT, surgical excision or without treatment ofthe primary tumor was expected. The lack of difference in tumor size can beexplained by differences of inflammatory response; thus, tumor proliferation andnecrosis. Although tumor growth curve did not differ between groups, themorphometric analysis showed significant differences on the VF of footpad cellularcomponents between surgically treated animals and animals treated with MB-PDTor without treatment of the primary tumor, as described in literature [7-9].

Surgically treated animals had higher tumor proliferation, less inflammatoryinfiltrate and a minor production of tumor necrosis. These results indicate thatanimals treated with surgical excision of the primary tumor did not develop acuteinflammation and the activation of innate immune system like that produced by PDT. On surgically treated animals there was no induction of adaptive immune responseagainst the treated tumor; therefore, tumor pro- 


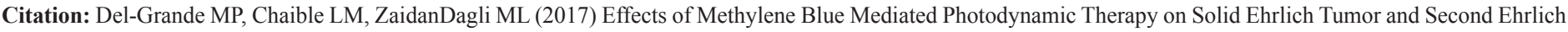
Tumor Implant in Mice. Arch Vet Sci Technol, 2017: AVST-122.

liferation was larger and occurswithout inducing important inflammation, unlike that observed on animals treatedwith PDT.

Relative spleen weight showed a significant difference between groups.Values were higher in MB-PDT and control groups, which may indicate a morestimulated immune system in those animals. Histopathology showed hyperplasia ofwhite pulp, confirming the suspicion of a greater immune stimulation in thesegroups. These results corroborate with Koberlik et al [4]who demonstratedthe role of spleen cells in the induction of tumor immunity produced by PDT throughtransferring spleen cells from animals previously treated with PDT toimmunosuppressed animals. In these animals, the PDT did not promote healing; however, when receiving spleen cells, the cure rates were back to the levels of theimmune competent animals. The analysis of the left popliteal lymph node, the lymphoid organ, revealed nosignificant statistical difference between relative weights on experimental groups.However, the significance level of $p=0.0622$ found is quite close to the valueconsidered to show significant difference among groups. Kousis et al. [10] found similar results when assessing by flow cytometry the cellular components oflymph node from animals treated with PDT. After PDT, the population of CD8+ Tlymphocytes in the regional lymph node was higher than in animals treated withsurgical excision of the tumor.

Histopathological analysis showed no differences on lymph node architecturewithin different groups. The presence of cells with phenotypic characteristics oftheEhrlich tumor were observed occupying the sub capsular space in all animalsinoculated on the footpad, as noted earlier by Dagli et al.[11]. The resultsobtained clearly show that animals treated with surgical excision of the primaryEhrlich tumor, when challenged with a new inoculation of tumor cells, presentdifferent local and systemic immune responses than animals treated with PDT or nottreated.The similarity of responses between animals treated with MB-PDT and thosereceiving no treatment of the primary tumor can be explained by the fact that theEhrlich tumor is immunogenic. As reported by Guerra (1983) [12] when animalsbearing Ehrlich solid tumor after 12 days of inoculation receive a second tumorchallenge, there is no progression of secondary tumor.Here, animals were treated after 9 days of primary tumor progression. Inanimals treated with surgical excision, the immune stimulation ceased before the 12days required to induceimmunity; therefore, we observed growth of secondarytumor and a poor activation of the immune system (seen in the analysis of lymphoidorgans and morphometry).

Animals treated with MB-PDT had a similar response to untreatedanimals. When compared to surgically treated animals, they showed less tumorproliferation and more inflammation andnecrosis of secondary tumor, in addition togreater systemic response seen in the analysis oflymphoid organs.In conclusion, the present study demonstrates that applying MB-PDT to aprimary Ehrlich tumor leads to a different inflammatory and cell death behavior of asecond Ehrlich tumor implant, possibly due to activation of adaptive immune system.

These results led us to think that MB-PDT can possibly impact the development ofmicro metastatic or metastatic growth in a variety of tumors. Therefore, biotechnological applications of MB-PDT, mainly for the production of cancervaccines, are currently under investigation.

\section{References}

1. Castano AP, Demidova TN, Hamblin MR (2004) Mechanisms in photodynamic therapy: part one - photosensitizers, photochemistry and cellular localization. PhotodiagnosisPhotodynTher 1: 279-293.

2. Tardivo JP, Del Giglio A, De Oliveira Cs, Gabrielli Ds, JunqueiraHc, et al. (2005) Methylene blue in photodynamic therapy: From basic mechanisms to clinical applications. PhotodiagnosisPhotodynTher 2: 175-191.

3. Gomer CJ,Ferrario A,Murphree AL (1987) The effect of localized porphyrin photodynamic therapy on the induction of tumour metastasis. Brit. J.Cancer 56: 27-32.

4. Korbelik M (1996) Induction of Tumor Immunity by Photodynamic Therapy. J. Clin Laser Med \&Surg 14: 329-334.

5. Castano AP, Mroz P, Hamblin MR (2006) Photodynamic therapy and antitumour immunity. Nat Rev Cancer 6: 535-545.

6. WeibelWR,Kistler GS, Scherle WF (1966) Practical stereological methods for morphometric cytology. J. Cell Biol30: 23-38.

7. Cubeddu R, Canti G, Musolino M, Pifferi A, Taroni P, et al. (1996) In vivo absorption spectrum of disulphonatedaluminiumphthalocyanine in a murine tumour 16 model. J. PhotochemPhotobiol34: 229-235.

8. Kabingu E, Vaughan L, Owczarczak B, Ramsey KD, Gollnick SO (2007) CD8 + T cell-mediated control of distant tumours following local photodynamic therapy is independent of CD4+ T cells and dependent on natural killer cells. Brit JCancer 96: 1839-1848.

9. Calin MA, Parasca SV (2006) Photodynamic therapy in oncology. J. OptoelectrAdv Mat 8: 1173-1179.

10. Kousis PC, HendersonBW, Maier PG, Gollnick SO (2007) Photodynamic therapy enhancement of antitumor immunity is regulated by neutrophils. Cancer Res 67: 10501-10510.

11. Dagli, Zaidan ML, Guerra, Luiz, Saldiva, et al. (1992) An experimental study on the lymphatic dissemination of the solid Ehrlich tumor in mice. Braz J Vet Res and a Sci 29: 97-103.

12. GuerraJL(1983) Aspectos do processoinflamatórioemcamundongosportadores de tumor de Ehrlich.79 f. Tese (DoutoradoemPatologia)Faculdade de MedicinaVeterinária e Zootecnia, Universidade de São Paulo, São Paulo. 